\title{
Visibilidad de la investigación contable colombiana en Google Académico
}

\author{
Hugo A. Macías \\ Profesor Asociado, Universidad de Medellín \\ Cómo citar: Macías, H. (2016) visibilidad de la investigación contable colombiana en google académico. Contexto 5; 3-6.
}

$\mathrm{E}$ $n$ el ambiente académico colombiano de los programas de Contaduría Pública, cada vez se pregunta por criterios más exigentes. A principios de la década pasada conseguir un profesor con maestría para un programa de Contaduría Pública, era toda una hazaña. Luego empezaron a preguntar por las acreditaciones, después por los registros calificados, más adelante por los grupos en Colciencias, por profesores con doctorado, algunos han empezado con acreditaciones internacionales, entre otros. Pero el indicador más difícil de conseguir en mitad de esta década son los artículos publicados en revistas Scopus y Web of Science (WoS, antes ISI). Como ese tipo de productos brillan por su ausencia (Macías, 2016), por ahora, Google Académico se ha convertido en la principal herramienta para medir el impacto de lo que hacemos en la academia contable nacional.

En el editorial de este quinto año, la revista Contexto, de la Universidad La Gran Colombia, Seccional Armenia, quiere abrir sus páginas a una reflexión sobre la integración de la academia contable colombiana y la visibilidad de esa construcción colectiva en Google Académico. Esta reflexión se da como continuidad a las propuestas del profesor Marlon David García Jiménez sobre la construcción de pensamiento y cultura académica en contabilidad (García, 2012), sobre la enseñanza de la contabilidad como una disciplina académica (García, 2014) y sobre el conocimiento científico en los profesores de Contaduría Pública (García, 2016). Concretamente, se trata de dar un paso adelante para mostrar que Google Académico, como herramienta, permite visibilizar los lazos construidos entre académicos, las revistas utilizadas como plataforma para la construcción de esos lazos, las características locales emergentes de la contabilidad como disciplina académica y la apropiación de esa construcción por parte de los profesores.

El profesor Marlon planteó en su trabajo de 2012 la necesidad de construir una cultura académica de la disciplina, ligando el objeto de estudio con los sujetos (García, 2012). Luego, él mismo resaltó que el nivel de formalidad del conocimiento contable exige que la contabilidad sea enseñada más como una disciplina científica que como un saber práctico (García, 2014). En el tercer artículo, apoyado ampliamente en la literatura nacional y con un trabajo empírico riguroso, basado en interacción formal con profesores, encontró que los académicos contables no son netamente empiristas, que vinculan el contenido de la ciencia con conceptos culturales, que valoran positivamente la teoría, los métodos científicos y entienden las limitaciones de la investigación (García, 2016). La interacción de los académicos contables entre sí y por medio de las publicaciones, es lo que permite construir colectivamente la contabilidad como disciplina académica conectada con las necesidades del entorno colombiano.
Para presentar esta dinámica, el documento tiene tres secciones principales: (1) el surgimiento de Google Académico como herramienta alternativa para medición de impacto; (2) su reciente uso en la academia contable anglosajona y (3) la academia contable colombiana vista desde Google Académico.

\section{Google Académico como herramienta alternativa de medición de impacto}

Los fundadores de Google.com, Larry Page y Sergey Brin, se conocieron en la Universidad de Stanford en 1995 y registraron la empresa en septiembre de 1997. Desde entonces la organización ha venido creciendo y diversificándose. Pero solo en noviembre de 2004 lanzaron Google Académico, luego de haberlo probado en Labs, una zona de experimentos de muchas de las funciones de Google. La herramienta se creó para realizar búsquedas de documentos especializados, como artículos, ponencias, libros, entre otros.

Google Académico irrumpió como una herramienta alternativa a los medidores de impacto de Scopus y WoS. Es decir, la principal fortaleza de esta herramienta, que la hace atractiva para la academia contable colombiana, es que en ella tienen visibilidad los trabajos que no hacen parte de las bases de datos privadas. La herramienta se considera alternativa en el sentido que beneficia a los autores que publican en fuentes que no están en WoS o Scopus, como libros, ponencias, revistas no estadounidenses $\mathrm{y}$, especialmente, las revistas de administración, negocios internacionales y contabilidad, que tienen baja cobertura en esos espacios; se considera que la herramienta tiene cierto nivel de "democratización" del conocimiento (Harzing \& van der Wal, 2008). Sin embargo, esa "democratización" es parcial, porque los documentos publicados en bases privadas conservan participación mayoritaria (Chan, Chan, Tong, \& Zhang, 2016).

Entre las ventajas de la herramienta, se destacan: (1) tiene en cuenta un período más largo de citaciones que otros indicadores similares; (2) permite calcular el índice " $h$ " para autores y revistas, un índice que no se deja alterar por algún articulo particular que eleve el número promedio de citas y (3) la disponibilidad gratuita proporciona a todos los académicos los datos sobre sus citas, independientemente de los medios financieros de la institución a la que pertenezcan (Harzing \& van der Wal, Google Scholar as a new source for citation analysis, 2008). Entre las ventajas, también está la cobertura de bases de datos de acceso abierto y bases de editor único (Neuhaus, Neuhaus, Ashe, \& Wrede, 2006).

De otro lado, también son conocidas un grupo de debilidades de la herramienta, aunque se vienen corrigiendo. Inicialmente, una debilidad importante fue la falta de cobertura de las bases de datos de ciencias 
sociales y humanidades, así como su sesgo hacia publicaciones en inglés (Neuhaus, Neuhaus, Ashe, \& Wrede, 2006). Más recientemente, se ha criticado la falta de control de calidad necesario para utilizarlo como herramienta bibliométrica (Aguillo, 2012) y la repetición de citas en documentos que están disponibles en varias fuentes (Rosenstreich \& Wooliscroft, 2009; Moed, Bar-Ilan, \& Halevi, 2016).

En los últimos años ha aumentado con rapidez la cobertura de disciplinas que tradicionalmente estaban mal representadas en Google Académico y se ha conseguido una cobertura exhaustiva y estable. Se ha encontrado que esta herramienta permite ofrecer una comparación entre disciplinas menos sesgada que los indicadores de WoS. El uso de Google Académico podría corregir la posición tradicionalmente desfavorecida de las ciencias sociales en el análisis de las citas (Harzing, 2013). Así mismo, se ha encontrado que al utilizarlo para construir ranking de programas académicos de contabilidad, se llega a resultados similares que utilizando otros métodos más tradicionales (Chan, Chan, Tong, \& Zhang, 2016).

El hecho de que Google Académico haya tomado la delantera como herramienta para medir impacto de las publicaciones está generando reacciones en otros conglomerados privados. Un ejemplo visible es Scopus, que está ampliando su cobertura y mejorando la calidad de sus datos, en relación con los primeros años. En 2014 puso en marcha un proyecto ambicioso de índice de citas en libros, para agregar en el corto plazo 75.000 libros a Scopus (Moed, Bar-Ilan, \& Halevi, 2016). Por lo pronto, Google Académico es la herramienta que permite analizar la dinámica de la academia contable colombiana de manera más amplia e incluyente.

\section{Uso de Google Académico en la literatura contable anglosajona}

Las citas obtenidas a través de Google Académico se han utilizado ampliamente en la literatura contable ya que tienen una cobertura más amplia que las herramientas de Scopus o WoS (Rosenstreich \& Wooliscroft, 2009). El principal uso ha sido la medición del impacto de publicaciones como Journal of Emerging Technologies in Accounting -JETA (O'Leary, 2011; Muehlmann, Chiu, \& Liu, 2015), los primeros 20 años de Advances in Management Accounting --AMA (Guffey, 2014), los primeros 15 años de Advances in Accounting Behavioral Research -AABR (Guffey, 2016), las seis principales revistas de educación contable (Sangster, Fogarty, Stoner, \& Marriott, 2015) y 23 revistas de finanzas (Xu, Chan, \& Chang, 2016). En todos los casos se presentan las características de esta herramienta, que la ubican por encima de herramientas alternativas.

Para el caso de la revista JETA, inicialmente se compararon los artículos más citados de la revista en dos años diferentes y se encontró que son un conjunto relativamente estable; además, los documentos más citados son más del $70 \%$ de las citas de la revista y normalmente con el tiempo las citas se concentran más en el mismo número de artículos (O’Leary, 2011). Esta es una información útil para los equipos editoriales de las revistas colombianas, en el sentido que puede ser un grupo pequeño de autores los que contribuyen al incremento en el impacto de la revista. Cuatro años después se publicó un artículo más amplio donde se evaluaron los primeros 10 años de JETA; allí se encontró que el numero de citas en Google Académico es mucho mayor al registrado en Scopus y que la diferencia aumenta con respecto a WoS (Muehlmann, Chiu, \& Liu, 2015). En esa segunda evaluación se concluye que Google Académico es la principal fuente para medir el impacto de una revista, por su mayor nivel de citación, y que $W o S$ y Scopus son complementarios.
El profesor norteamericano Daryl Guffey ha llevado a cabo varios estudios sobre el impacto de publicaciones contables, utilizando Google Académico como herramienta principal. En uno de ellos evaluó el impacto de los primeros 20 años de AMA, midiendo las citas de cada autor y de toda la revista, tanto de manera anual como a lo largo del período completo (Guffey, 2014). Más adelante, evaluó los primeros 15 años de AABR, donde identificó los documentos, autores, facultades y programas de doctorado que más contribuyeron al desarrollo de esta publicación que desarrolla un enfoque particular de la contabilidad (Guffey, 2016). En ambos casos presentó un mapa de relaciones entre la comunidad que interactúa en estas dos publicaciones.

En otros trabajos se evalúan grupos de revistas, utilizando la misma herramienta. En uno de los casos se midió el impacto de las seis principales revistas de educación contable, publicadas en inglés; allí se identificaron las revistas externas donde son citadas estas revistas, se hicieron recomendaciones para los autores que quieren aumentar su impacto y se identificaron temas de preocupación para los editores (Sangster, Fogarty, Stoner, \& Marriott, 2015). En otro trabajo, se evaluaron 23 revistas de finanzas de corriente principal, entre 1990 y 2010; el principal objetivo fue construir diferentes rankings donde prevalecieron instituciones estadounidenses, pero también se identificaron avances recientes en instituciones europeas, de Asia y del Pacífico (Xu, Chan, \& Chang, 2016). En este último estudio también se indicó que Google Scholar tiene mejores características para medir el impacto que las herramientas de Scopus y WoS.

Como puede verse, a pesar de que ha transcurrido solo una década desde el lanzamiento de Google Académico y que ha estado en constante evolución, la literatura contable no solo la ha incorporado para medir el impacto de sus autores y de sus publicaciones, sino que la considera una herramienta con ventajas sobre otras similares de conglomerados privados como Scopus y WoS. De todas maneras, "no hay una sola medida perfecta del impacto de la investigación académica. Otras medidas son necesarias para captar el valor de la investigación y/o el impacto en audiencias más amplias" (Muehlmann, Chiu, \& Liu, 2015, p. 39).

\section{Revistas y académicos contables colombianos en Google Académico}

Un número bajo de académicos contables colombianos ha creado el perfil de Google Académico. Dentro de los que sí lo tienen, se destaca el profesor Mauricio Gómez Villegas (Universidad Nacional de Colombia) quien registra el mayor impacto académico, medido con esta herramienta. Lejos de él, pero también con un impacto alto, aparecen en su orden profesores como, Jaime Andrés Correa (Universidad de Antioquia), Jorge Alberto Rivera Godoy (Universidad del Valle), Rafael Franco Ruiz (Universidad Libre de Colombia), Jair Albeiro Osorio (Universidad de Antioquia), Gabriel Rueda Delgado (Universidad Nacional de Colombia), Marco Antonio Machado (Universidad de Antioquia), Carlos Eduardo Castaño (Universidad de Antioquia), Hugo A. Macías (Universidad de Medellín), Alejandra Patiño (Universidad Santo Tomás), Fernando Chaparro García (Universidad Autónoma de Bucaramanga), Edison Fredy León Paime (Universidad Jorge Tadeo Lozano), Abel Cano Morales (Universidad de Medellín), Fabián Leonardo Quinche Martín (Universidad la Gran Colombia) y Elkin Quirós (Universidad Católica Luis Amigó y Universidad Autónoma Latinoamericana).

Los artículos más citados en Google Académico de estos 15 autores dan una señal clara sobre cuáles son las fuentes más utilizadas en las 
discusiones de la academia contable colombiana. De los 75 documentos más citados (cinco de cada autor), 11 son libros, 11 son artículos publicados en Cuadernos de Contabilidad, de la Pontificia Universidad Javeriana y 10 son artículos publicados en la Revista Facultad de Ciencias Económicas: Investigación y Reflexión, de la Universidad Militar Nueva Granada. Esto muestra que los libros locales siguen siendo una fuente importante de consulta y que estas dos revistas han concentrado la atención de las discusiones nacionales. Las dos revistas fueron editadas por un período prolongado y posicionadas en este lugar de liderazgo, por los profesores Gabriel Rueda Delgado y Fredy León Paime, respectivamente.

Luego de los libros y las dos revistas ya mencionadas, está la revista Contaduría Universidad de Antioquia, donde se publicaron 7 de los 75 trabajos más citados. Esta revista es la más antigua en la academia contable colombiana, ha pasado por varios períodos desde su creación en 1982 (Macías \& Patiño, 2014) y conserva una posición de liderazgo dentro de la academia contable nacional. Muy cerca de allí se ubican la revista Innovar, la revista Lúmina y la Revista Internacional Legis de Contabilidad y Auditoría, con 4 de los trabajos más citados cada una. La revista Innovar se ha destacado por ser la revista colombiana de ciencias económicas que ha alcanzado el mayor nivel en los ranking internacionales. La revista Lúmina fue la primera revista especializada en contabilidad que alcanzó la indexación de Colciencias. Por su parte, la revista de la empresa Legis ha ganado y mantenido un lugar de alta aceptación entre los académicos contables y sobre ella ya se han hecho análisis bibliométricos (Santos, 2012). Las citas de Google Académico evidencian que estas cuatro revistas también están en el centro de las discusiones contables en Colombia, independiente de la clasificación que Colciencias o alguna otra herramienta de medición hagan de ellas.

Una parte de las discusiones contables se da por fuera de las revistas especializadas en contabilidad, según lo muestran las citaciones en Google Académico. En ese grupo aparecen la revista Semestre Económico (3 artículos) y las revistas Cuadernos de Administración, Estudios Gerenciales y Perfil de Coyuntura Económica, con 2 artículos cada una; otras revistas tienen una participación menor. Si bien hay diferencias claras en los perfiles de los 15 profesores a los que se hace referencia en este trabajo, quien tiene un perfil más diferenciable es el profesor Fernando Chaparro García, quien ha publicado cuatro de sus cinco principales trabajos en revistas extranjeras, tres de ellos en inglés. Para cuatro de estos profesores su trabajo más citado es un libro (Rafael Franco, Jair Osorio, Marco Machado y Elkin Quiroz), pero para los demás su trabajo de mayor impacto es un artículo escrito en español.

Si bien estos datos presentados son solo una aproximación a la dinámica de la academia contable colombiana, si dan una idea de cuáles son los espacios donde en Colombia se intenta hacer una construcción colectiva de conocimiento contable. Nótese que ninguno de los autores mencionados tiene una ponencia entre sus trabajos de mayor impacto, medido con Google Académico. Esto es importante, en la medida en que la mayor parte de la producción académica colombiana está en ponencias y documentos que no circulan entre la comunidad (Patiño, Romero, \& Jara, 2010). En este sentido es necesario hacer una invitación a los organizadores de los múltiples eventos contables, para que las memorias se publiquen en las revistas académicas y no en discos compactos o memorias USB. Los editores de revistas necesitan trabajos de calidad, pero buena parte de la producción académica queda sepultada en dispositivos que no consultan ni los asistentes a los eventos.

Es importante señalar que en Colombia hay un grupo de académicos contables que se resiste a aceptar las citas como indicador de calidad o de impacto de los trabajos publicados, como pudo evidenciarse en un debate escrito desarrollado tres años atrás (Macías, 2013a). La posición que se defiende aquí es que un bajo nivel de citaciones refleja ausencia de construcción colectiva, desprecio por lo que se ha construido previamente y descontextualización de los nuevos documentos. Si bien hay muchas críticas válidas con respecto a las citas, herramientas como Google Académico nos permiten visibilizar las interrelaciones que estamos construyendo, los liderazgos tradicionales y emergentes que tanto cuesta reconocer y avanzar hacia una integración, hacia lo que el profesor Marlon García llama construcción de pensamiento y cultura académica en contabilidad.

Además del sentido que tienen las citas, como evidencia de construcción colectiva, ahora Colciencias clasificará las revistas colombianas que aún no han ingresado a $W o S$ o Scopus, según el número de citas que sus artículos registren en Google Académico (Macías, 2016). En el caso de los autores, mientras emprendemos el camino de publicación internacional que ya tomaron los colegas contables de Brasil, México y otros países de la región (Macías, 2013b), crear los perfiles de Google Académico y consultar los de los colegas, nos dará señales de caminos para avanzar. Son más de 500 los investigadores colombianos vinculados a los grupos de investigación contable (Patiño, Romero, \& Jara, 2010), pero un número bajo de ellos está utilizando estas herramientas de medición de impacto.

Para finalizar, vale la pena recomendar a los académicos contables colombianos crear su perfil en Google Académico. Al hacerlo, tendrán entre otros los siguientes beneficios: (1) Conocer cuántas citas tiene cada trabajo publicado, quién lo ha citado y qué citan de él; (2) tomar decisiones sobre trabajos futuros, en términos de temas, enfoques, métodos, coautorías, espacios de publicación y divulgación posterior a la publicación; (3) presentar información sintética y clara, de acceso público, para otros académicos que quieran hacer seguimiento a los trabajos propios; (4) acceder, mediante un solo click, al ranking de autores para cada una de las líneas de investigación desarrolladas y acceder a los perfiles de ellos; (5) acceder, mediante un solo click, al ranking de autores que trabajan en la misma institución y a sus perfiles. Mientras conseguimos participar en las discusiones internacionales, como lo hacen cientos de académicos contables desde el mundo en desarrollo en diferentes regiones del planeta, podemos conocer detalles de nuestras construcciones colectivas, acudiendo a los indicadores de Google Académico.

\section{Referencias bibliográficas}

Aguillo, I. F. (2012). Is Google Scholar useful for bibliometrics? A webometric analysis. Scientometrics , 91 (2), 343-351.

Chan, J. Y., Chan, K., Tong, J. Y., \& Zhang, F. (2016). Using Google Scholar citations to rank accounting programs: a global perspective. Review of Quantitative Finance and Accounting , 47 (1), 29-55.

García, M. D. (2014). Enseñanza de la contabilidad como disciplina académica: Concepciones de ciencias del profesorado y pensamiento crítico. Entramado, 10 (1), 164-174.

García, M. D. (2012). Lenguaje pedagógico, filosofía e investigación: Relación con la construcción de pensamiento y cultura académica en contabilidad. Revista Lúmina (13), 152-167.

García, M. D. (2016). Modelos de conocimiento científio escolar en un grupo de maestros de programas universitarios de Contaduría Pública. Sophia, 12 (1), 85-105. 
Guffey, D. M. (2016). An analysis of the research contributions of advances in accounting behavioral research: 1998-2012. Advances in Accounting Behavioral Research, 19, 1-32.

Guffey, D. M. (2014). Citation analysis of advances in management accounting: The first 20 volumes. Advances in Management Accounting , 23, 45-64.

Harzing, A. K. (2013). A preliminary test of Google Scholar as a source for citation data: A longitudinal study of Nobel prize winners. Scientometrics , 94 (3), 1057-1075.

Harzing, A. K., \& van der Wal, R. (2008). Google Scholar as a new source for citation analysis. Ethics in Science and Environmental Politics , $8(1), 61-73$.

Macías, H. A. (2013a). Debate sobre el presente y el futuro de las revistas contables colombianas: acuerdos, desacuerdos y retos. Cuadernos de contabilidad, 14 (34), 265-271.

Macías, H. A. (2013b). Internacionalización de las revistas contables: un aporte al debate polifónico. Cuadernos de Contabilidad , 14 (35), 785-794.

Macías, H. A. (2016). La investigación contable colombiana frente a los nuevos criterios de medición de Colciencias. En-Contexto $4(4), 39-48$.

Macías, H. A., \& Patiño, R. A. (2014). Evolución de las revistas contables colombianas: de la reflexión a la investigación. Contaduría Universidad de Antioquia (64), 13-48.

Moed, H. F., Bar-Ilan, J., \& Halevi, G. (2016). A new methodology for comparing Google Scholar and Scopus. Journal of Informetrics , 10 (2), 533-551.
Muehlmann, B. W., Chiu, V., \& Liu, Q. (2015). Emerging technologies research in accounting: JETA's first decade. Journal of Emerging Technologies in Accounting , 12 (1), 17-50.

Neuhaus, C. F., Neuhaus, E. E., Ashe, A., \& Wrede, C. (2006). The depth and breadth of google scholar: An empirical study. Portal, $6(2), 127-141$

O'Leary, D. E. (2011). The ten most-cited papers in Journal of Emerging Technologies in Accounting: 2009 versus 2011. Journal of Emerging Technologies in Accounting , 8 (1), 1-5.

Patiño, R. A., Romero, A., \& Jara, K. G. (2010). Características de los investigadores relacionados con programas de contaduría pública y con temáticas relacionadas. Cuadernos de Contabilidad, 11 (28), 171-199.

Rosenstreich, D., \& Wooliscroft, B. (2009). Measuring the impact of accounting journals using Google Scholar and the g-index. British Accounting Review , 41 (4), 227-239.

Sangster, A., Fogarty, T., Stoner, G., \& Marriott, N. (2015). The Impact of Accounting Education Research. Accounting Education, 24 (5), 423-444

Santos, G. (2012). Aproximaciones a un análisis bibliométrico de la Revista Internacional Legis de Contabilidad \& Auditoría 2000-2012. Revista Internacional Legis de Contabilidad \& Auditoría , 15 (49), 183-226.

Xu, N., Chan, K. C., \& Chang, C. H. (2016). A quality-based global assessment of financial research. Review of Quantitative Finance and Accounting , 46 (3), 605-631. 